\title{
Noncardiac genetic predisposition in sudden infant death syndrome
}

\author{
Belinda Gray, MBBS PhD, Elijah R. Behr, MD et al."
}

Purpose: Sudden infant death syndrome (SIDS) is the commonest cause of sudden death of an infant; however, the genetic basis remains poorly understood. We aimed to identify noncardiac genes underpinning SIDS and determine their prevalence compared with ethnically matched controls.

Methods: Using exome sequencing we assessed the yield of ultrarare nonsynonymous variants (minor allele frequency [MAF] $\leq 0.00005$, dominant model; MAF $\leq 0.01$, recessive model) in 278 European SIDS cases (62\% male; average age $=2.7 \pm 2$ months) versus 973 European controls across 61 noncardiac SIDSsusceptibility genes. The variants were classified according to American College of Medical Genetics and Genomics criteria. Casecontrol, gene-collapsing analysis was performed in eight candidate biological pathways previously implicated in SIDS pathogenesis.

Results: Overall 43/278 SIDS cases harbored an ultrarare singlenucleotide variant compared with $114 / 973$ controls (15.5 vs. $11.7 \%$, $p=0.10$ ). Only $2 / 61$ noncardiac genes were significantly overrepresented in cases compared with controls (ECE1, 3/278 [1\%] vs. $1 / 973[0.1 \%] p=0.036 ;$ SLC6A4, 2/278 [0.7\%] vs. $1 / 973[0.1 \%]$ $p=0.049$ ). There was no difference in yield of pathogenic or likely pathogenic variants between cases and controls (1/278 [0.36\%] vs. 4/ $973[0.41 \%] ; p=1.0)$. Gene-collapsing analysis did not identify any specific biological pathways to be significantly associated with SIDS.

Conclusions: A monogenic basis for SIDS amongst the previously implicated noncardiac genes and their encoded biological pathways is negligible.

Genetics in Medicine (2019) 21:641-649; https://doi.org/10.1038/s41436018-0131-4

Keywords: genetics; molecular autopsy; sudden infant death syndrome; exome sequencing

\section{INTRODUCTION}

Sudden infant death syndrome (SIDS) is defined as "the sudden death of an infant under 1 year of age which remains unexplained after thorough investigation including detailed clinical and pathological review."1,2 The peak incidence occurs between 2 and 4 months of age and has been often associated with environmental risk factors such as prone sleep position and maternal smoking. ${ }^{3}$ Despite successful targeted risk reduction campaigns such as the "back-to-sleep" campaigns in the 1990s, SIDS remains a leading cause of sudden infant death, occurring at a rate of 27-38/100,000 live births in the United Kingdom and the United States respectively. ${ }^{3-5}$

Research in SIDS has proposed that unexplained infant deaths result from "abnormalities at birth that make them vulnerable to potential life-threatening challenges in infancy." The "triple-risk hypothesis" proposed the convergence of three overlapping factors: (1) a "vulnerable" infant, (2) a critical development period, and (3) an exogenous stressor. ${ }^{7}$ Accordingly, SIDS does not typically occur in normal infants, but rather, in vulnerable infants with an underlying abnormality. ${ }^{3}$

Rather than a single etiology underlying the majority of infant vulnerability, SIDS may be due to multiple distinct genetic disorders with a common final endpoint of sudden death occurring during sleep. ${ }^{8}$ Several studies have implicated both common and rare genetic variants within genes involved in several biological pathways including neurological conditions, neuronal signaling, inborn errors of metabolism, respiratory control, musculoskeletal conditions, immune response, and genetic heart disease (GHD) as a basis for underlying infant vulnerability. ${ }^{2,9-14}$

Using exome sequencing and a targeted analysis of 90 GHD-susceptibility genes in over 400 SIDS cases, we determined recently a $5 \%$ prevalence of GHD-associated "pathogenic" or "likely pathogenic" variants as a potential monogenic basis for SIDS. ${ }^{15}$ There was only an excess burden of rare variants in the major channelopathy genes when nearly 300 Caucasian cases were compared with approximately 1000 Caucasian controls.

Correspondence: Michael J. Ackerman (ackerman.michael@mayo.edu) or Elijah R. Behr (ebehr@sgul.ac.uk). "A full list of authors and their affliations appears at the end of the paper.

Co-Equal First Authors: Belinda Gray, David J. Tester.

Joint and Co-Corresponding Last Authors: Michael A. Simpson, Michael J. Ackerman, Elijah R. Behr.

Submitted 18 April 2018; accepted: 28 June 2018

Published online: 24 August 2018 
Here, we conducted a SIDS-susceptibility variant analysis in the same cohort examining the previously published, noncardiac SIDS-susceptibility genes followed by a genecollapsing rare variant burden analysis involving multiple noncardiac, biological pathways implicated previously in SIDS pathogenesis.

\section{Study population}

\section{MATERIALS AND METHODS}

As previously described, the SIDS cohort $(N=427)$ consisted of 95 coroners' cases from the United Kingdom (UK; London, Sheffield, Edinburgh, and Bristol) and 332 coroner/medical examiner/forensic pathologist-referred cases collected from six ethnically and geographically diverse United States (US) populations. $^{15}$ Enrollment criteria included (1) sudden unexplained death of an infant $<1$ year of age, (2) reported European descent, and (3) a comprehensive negative medicolegal autopsy including a negative toxicology screen and death scene investigation. Infants with asphyxia or specific disease causing death were excluded. Ethnicity was self-reported by the referring coroner/medical examiner. This study complies with the Declaration of Helsinki; locally appointed ethics committees including Mayo Clinic's Institutional Review Board have approved the research protocol.

\section{Control population}

A total of 973 control exomes (509 females, 464 males) from the ICR1000 UK exome series and the 1958 Birth Cohort study were included for analysis. ${ }^{16}$ As previously reported, exome sequencing was performed using the Illumina TruSeq and Illumina instruments. ${ }^{16}$

\section{Exome sequencing}

As previously described, genomic DNA isolated from each SIDS case underwent exome sequencing at the KCL-GSTT Biomedical Research Centre Genomics Platform, London, UK or Mayo Clinic's Medical Genome Facility, Rochester, Minnesota. To avoid potential confounding due to population stratification resulting from genetic admixture, a principal component analysis (PCA) was performed as previously described. ${ }^{15}$ Furthermore, quality control metrics excluded seven cases due to insufficient exome coverage and one individual from a half-sibling pair. A case-control data set was established for 278 SIDS cases (confirmed as Caucasian by PCA) and 973 European controls. Detailed methodology can be found in the Online Supplement.

\section{Case-control noncardiac SIDS-susceptibility gene specific variant analysis}

A list of 55 SIDS-susceptibility genes involving multiple, noncardiac biological pathways implicated previously in SIDS pathogenesis was derived from Salomonis' integrated mechanism review article, "Systems-level perspective of sudden infant death syndrome," published in 2014 (ref. ${ }^{8}$ ). This literature review-based list included genes with sufficient evidence for involvement of SIDS based on the reported conclusions of manuscript authors. ${ }^{8}$ Based on our own literature search of articles from 2014 to 2018, 6 additional SIDS-susceptibility genes were included for a total list of 61 noncardiac candidate genes (see Online Supplement Table 1) (ref.$^{17-21}$ ).

Following exome sequencing, single-nucleotide variants (SNVs) and insertion/deletions (INDELs) were filtered to identify variants which followed either a dominant or recessive inheritance pattern using Ingenuity Variant Software (Qiagen, Redwood City, CA). All variants within the 61 noncardiac SIDS-susceptibility genes were first filtered for a call quality score $\geq 20$ and a read depth $\geq 10$. Only nonsynonymous (NSV, i.e., amino acid altering: missense, nonsense, splice-error, frame-shift INDEL, or in-frame INDEL) were considered potentially pathogenic. For the dominant model, only ultrarare variants (minor allele frequency $[\mathrm{MAF}$ ] $\leq 0.00005$ (1: 20,000 alleles) in Genome Aggregation Database (gnomAD; http://gnomad. broadinstitute.org) were considered. Variants with a MAF > 0.00005 in any ethnic group of gnomAD were excluded, unless observed only once in that ethnic group. For the recessive inheritance model, only rare $(\mathrm{MAF} \leq 0.01$ in gnomAD) variants present as either homozygotes or compound heterozygotes (two unique pathogenic variants in the same gene) were included. Importantly, for compound heterozygotes, it was assumed that the variants were present in trans; however, parental DNA was unavailable to confirm this. Variants with a homozygous frequency $>0.0001$ in gnomAD were excluded from analysis. A comparison of yield of NSVs for both the dominant and recessive model was performed for all 61 noncardiac SIDS-susceptibility genes.

The American College of Medical Genetics and Genomics (ACMG) and Association for Molecular Pathology (AMP) standards and guidelines for the interpretation of sequence variants was used to further assist in the interpretation and annotation of our genetic findings. ${ }^{22}$ Automatic variant classification was performed using InterVar; a freely available web-based bioinformatics software tool for clinical interpretation of genetic variants by the ACMG/AMP 2015 guideline. $^{23}$

\section{SIDS candidate biological pathway gene-collapsing analysis} We identified previously a list of 90 genetic heart disease (GHD)-associated genes. ${ }^{15}$ Using PubMed as our search engine, with the key phrase of "sudden infant death" plus "gene," "polymorphism," or "mutation," and OMIM, with the key words of "sudden infant death," "epilepsy," and "inborn errors of metabolism," an additional list of 241 noncardiac genes were identified for a gene-collapsing rare variant burden analysis. Only population-based SIDS cohorts, case reports, and literature reviews between 1990 and 2016 were used. Studies based on definitions of SIDS contrary to current practices were excluded.

We performed case-control, gene-collapsing analyses of ultrarare (MAF <0.00005), NSVs with a combined annotation dependent depletion (CADD) score $\geq 20$ in candidate biological pathways previously implicated in SIDS 
pathogenesis. A CADD score $\geq 20$ is equivalent to a 0.99 probability that the variant has a functional impact. ${ }^{24}$ The unit of analysis was a collection of genes from each pathway (See Online Supplement Table 2): GHD (90 genes), epilepsy (72 genes), inborn errors of metabolism (69 genes), other neurological (33 genes), respiratory system (37 genes), autonomic nervous system (13 genes), immune system (12 genes), and nicotine response ( 3 genes).

\section{Statistics}

Categorical variables were expressed as absolute numbers and percentage, and compared with Fisher's exact or chi-square tests. Probability values were based on two-sided tests considered significant at $P<0.05$. For this exploratory analysis, a Bonferroni correction of $p<0.0008$ (0.05 divided by 61) was not applied. Analysis was conducted with SPSS version 18.0 software (SPSS Chicago III).

\section{Demographics}

\section{RESULTS}

The case cohort consisted of 278 European SIDS cases (173 males, 105 females; average age $=2.7 \pm 1.98$ months), described previously by our group. ${ }^{15}$ The epidemiologically high-risk age group of 2-4 months (55.4\%) and male gender (62.2\%) accounted for the majority of the cases. Sleep characteristics were known in $60 \%$ of the cohort, of whom $66 / 172(38 \%)$ were cosleeping at the time of the SIDS death (Table 1).

SIDS-susceptibility gene-specific analysis of the noncardiac genes previously implicated in SIDS

Considering a dominant inheritance model, a total of 44 unique (42 novel), ultrarare NSVs (20 missense, 2 in-frame

Table 1 Summary of the sudden infant death syndrome cohort demographics

\begin{tabular}{lll} 
Demographics & & $\begin{array}{l}\text { European ancestry } \\
(n=278)\end{array}$ \\
\hline Sex & Male & $173(62.2 \%)$ \\
Age & Female & $105(37.8 \%)$ \\
Age group & Average (months) & $2.7 \pm 1.98$ \\
& Range (months) & $0.1-12$ \\
& $<2$ months & $81(29.1 \%)$ \\
Sleep position & $2-4$ months & $154(55.4 \%)$ \\
& $>4$ months & $43(14.7 \%)$ \\
& Supine & $85(30.6 \%)$ \\
& Prone & $52(18.7 \%)$ \\
& Side & $29(10.4 \%)$ \\
Cosleeping & Seated & $2(0.72 \%)$ \\
& Unknown & $110(39.6 \%)$ \\
& Yes & $66(23.7 \%)$ \\
& No & $106(38.1 \%)$ \\
\hline
\end{tabular}

Values are $n(\%)$ or mean \pm SD deletions, 1 frame-shift deletion, and 1 stop-loss) were identified in 43/278 (15.5\%) SIDS cases overall (Fig. 1). Further, 2/278 (0.72\%) SIDS cases hosted $>1$ ultrarare NSV. In comparison, a total of 115 unique (104 novel), ultrarare NSVs (109 missense, 1 in-frame deletion, 2 frame-shift deletions, 1 start-loss, and 1 stop-loss) were identified in $114 / 973(11.7 \%, p=0.10)$ European controls (Fig. 1). Further, 6/973 (0.62\%) European controls hosted $>1$, ultrarare NSV.

The gene-specific yields for the SIDS cohort and the European controls are shown in Table 2. For 59 of the 61 genes, there was no overrepresentation of ultrarare NSVs in SIDS cases versus controls at even the low stringent $p<0.05$ threshold (Table 2). Two genes hosted more ultrarare NSVs in SIDS cases than controls at this threshold: ECE1 (endothelin-converting enzyme, [3/278 (1.1\%) cases vs. $1 / 973(0.1 \%)$ controls; $p=0.036]$ ) and SLC6A4 (solute carrier family 6 member 4 , also known as the serotonin transporter 1 [2/278 (0.7\%) cases vs. 0/973 (0\%) controls; $p=0.049])$ (Table 2).

Following variant classification using the strict ACMG guidelines, 1 of the 44 (2.2\%) SIDS case variants and 3 of the 115 (3.5\%) European control variants, achieved either a "pathogenic" or "likely pathogenic" designation. All other variants were classified as variants of uncertain significance (VUS). There was no difference in overall yield of pathogenic or likely pathogenic variants between the European SIDS and control cohorts (1/278 [0.36\%] vs. $3 / 973$ [0.31\%]; $p=1.0$; see Online Supplement Tables 3 and 4).

A heterozygous pathogenic p.V153fs ${ }^{\star} 41$-SLC22A5 variant was identified in a 4-month-old female SIDS case. The p.V153fs ${ }^{*} 41-S L C 22 A 5$ variant has been observed previously in patients with primary carnitine deficiency, an autosomal

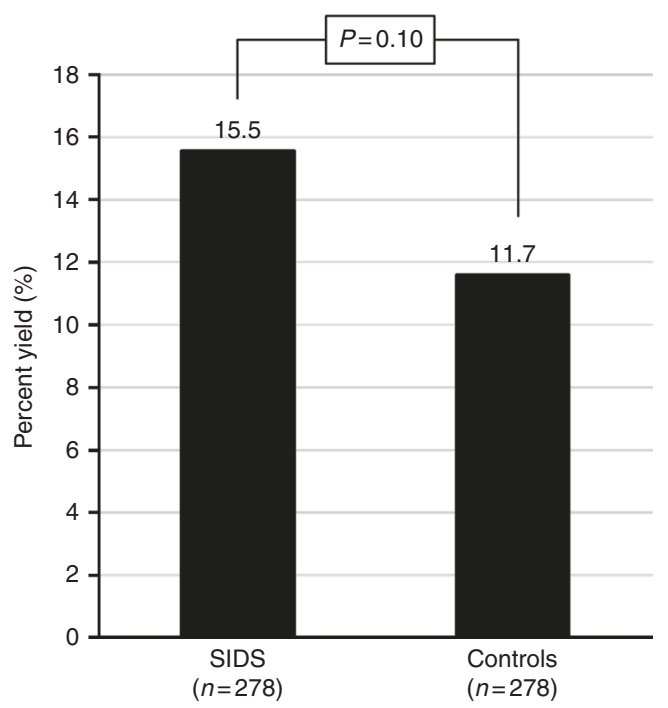

Fig. 1 Yield of ultrarare nonsynonymous variants in previously published, noncardiac SIDS-susceptibility genes. Bar graph depicting the percent yield of ultrarare (minor allele frequency $<0.00005$ ), nonsynonymous variants identified among the 61 noncardiac SIDS-susceptibility genes for the SIDS case and European control cohorts. SIDS, sudden infant death syndrome. 
Table 2 Gene-specific yield of ultrarare nonsynonymous variants in cases and controls

\begin{tabular}{|c|c|c|c|c|c|}
\hline Gene & $\begin{array}{l}\text { Cases } \\
(n=278)\end{array}$ & Percent cases & $\begin{array}{l}\text { Controls } \\
(n=973)\end{array}$ & Percent controls & $p$ value \\
\hline ECE1 & 3 & 1.1 & 1 & 0.1 & 0.036 \\
\hline SLC6A4 & 2 & 0.7 & 0 & 0.0 & 0.049 \\
\hline CHRNB4 & 2 & 0.7 & 2 & 0.2 & 0.216 \\
\hline NOS1AP & 2 & 0.7 & 2 & 0.2 & 0.216 \\
\hline SLC9A3 & 2 & 0.7 & 2 & 0.2 & 0.216 \\
\hline FEV & 1 & 0.4 & 0 & 0.0 & 0.220 \\
\hline HSPD1 & 1 & 0.4 & 0 & 0.0 & 0.220 \\
\hline HTR1A & 1 & 0.4 & 0 & 0.0 & 0.220 \\
\hline$\angle L 1 B$ & 1 & 0.4 & 0 & 0.0 & 0.220 \\
\hline MBL2 & 1 & 0.4 & 0 & 0.0 & 0.220 \\
\hline$T L X 3$ & 1 & 0.4 & 0 & 0.0 & 0.220 \\
\hline RET & 2 & 0.7 & 3 & 0.3 & 0.309 \\
\hline SLC22A5 & 2 & 0.7 & 3 & 0.3 & 0.309 \\
\hline HTR3A & 0 & 0.0 & 6 & 0.6 & 0.348 \\
\hline ACADS & 1 & 0.4 & 1 & 0.1 & 0.395 \\
\hline NTRK2 & 1 & 0.4 & 1 & 0.1 & 0.395 \\
\hline SST & 1 & 0.4 & 1 & 0.1 & 0.395 \\
\hline AQP4 & 1 & 0.4 & 2 & 0.2 & 0.530 \\
\hline$C L C N K B$ & 1 & 0.4 & 2 & 0.2 & 0.530 \\
\hline GCK & 1 & 0.4 & 2 & 0.2 & 0.530 \\
\hline IL6R & 1 & 0.4 & 2 & 0.2 & 0.530 \\
\hline $1 L 13$ & 0 & 0.0 & 4 & 0.4 & 0.581 \\
\hline MAP2 & 5 & 1.8 & 14 & 1.4 & 0.589 \\
\hline CHAT & 0 & 0.0 & 5 & 0.5 & 0.592 \\
\hline GRIN1 & 0 & 0.0 & 5 & 0.5 & 0.592 \\
\hline CHRNB2 & 2 & 0.7 & 4 & 0.4 & 0.620 \\
\hline OPRM1 & 2 & 0.7 & 4 & 0.4 & 0.620 \\
\hline ACADM & 0 & 0.0 & 1 & 0.1 & 1.000 \\
\hline ADCYAP1 & 0 & 0.0 & 1 & 0.1 & 1.000 \\
\hline BDNF & 0 & 0.0 & 1 & 0.1 & 1.000 \\
\hline C4A & 0 & 0.0 & 0 & 0.0 & 1.000 \\
\hline$C 4 B$ & 0 & 0.0 & 0 & 0.0 & 1.000 \\
\hline CASP3 & 0 & 0.0 & 0 & 0.0 & 1.000 \\
\hline CHRM2 & 0 & 0.0 & 1 & 0.1 & 1.000 \\
\hline CHRNA4 & 0 & 0.0 & 3 & 0.3 & 1.000 \\
\hline CHRNA7 & 0 & 0.0 & 1 & 0.1 & 1.000 \\
\hline CPT1A & 1 & 0.4 & 4 & 0.4 & 1.000 \\
\hline CPT2 & 2 & 0.7 & 6 & 0.6 & 1.000 \\
\hline EN1 & 0 & 0.0 & 0 & 0.0 & 1.000 \\
\hline FMO3 & 0 & 0.0 & 1 & 0.1 & 1.000 \\
\hline G6PC & 1 & 0.4 & 3 & 0.3 & 1.000 \\
\hline GABRA1 & 0 & 0.0 & 1 & 0.1 & 1.000 \\
\hline GNB3 & 1 & 0.4 & 3 & 0.3 & 1.000 \\
\hline HADHA & 2 & 0.7 & 9 & 0.9 & 1.000 \\
\hline$H A D H B$ & 0 & 0.0 & 0 & 0.0 & 1.000 \\
\hline IL10 & 0 & 0.0 & 0 & 0.0 & 1.000 \\
\hline IL1A & 0 & 0.0 & 0 & 0.0 & 1.000 \\
\hline ILIRN & 0 & 0.0 & 1 & 0.1 & 1.000 \\
\hline 126 & 0 & 0.0 & 0 & 0.0 & 1.000 \\
\hline IL8 (CXCL8) & 0 & 0.0 & 3 & 0.3 & 1.000 \\
\hline$\angle M X 1 B$ & 0 & 0.0 & 0 & 0.0 & 1.000 \\
\hline
\end{tabular}


Table 2 continued

\begin{tabular}{lllllc}
\hline Gene & $\begin{array}{l}\text { Cases } \\
(n=278)\end{array}$ & Percent cases & $\begin{array}{l}\text { Controls } \\
(n=973)\end{array}$ & Percent controls value \\
\hline MAOA & 0 & 0.0 & 3 & 0.3 & 1.000 \\
PHOX2A & 0 & 0.0 & 0 & 0.0 & 1.000 \\
PHOX2B & 0 & 0.0 & 0 & 0.0 & 1.000 \\
SULT1A1 & 1 & 0.4 & 3 & 0.3 & 1.000 \\
TAC1 & 0 & 0.0 & 0 & 0.0 & 1.000 \\
TH & 0 & 0.0 & 3 & 0.3 & 1.000 \\
TNF & 0 & 0.0 & 0 & 0.0 & 1.000 \\
TPH2 & 0 & 0.0 & 2 & 0.2 & 1.000 \\
TSPYL1 & 0 & 0.0 & 2 & 0.2 & 1.000 \\
VEGFA & 0 & 0.0 & 0 & 0.0 \\
\hline G & 0 & 0.000 & \\
\hline
\end{tabular}

Genes are listed in order by $p$ value. A $p$ value $<0.05$ was considered potentially significant.

recessive disorder of the carnitine cycle resulting in defective fatty acid oxidation. ${ }^{25}$ However, because a second SLC22A5 variant was not identified in this SIDS case, it is unlikely this infant had undiagnosed primary carnitine deficiency. Two controls also hosted ultrarare likely pathogenic heterozygous variants (p.Y447C-SLC22A5, and p.G827R-GRIN1). Interestingly, the p.G827R-GRIN1 variant has also been identified previously as a de novo heterozygous variant in three unrelated individuals with severe intellectual disability, movement disorder, and seizures. ${ }^{26,27}$

Considering a recessive inheritance model, homozygous or compound heterozygous variants were observed in $2 / 278$ (0.72\%) SIDS cases compared with $3 / 973(0.31 \%)$ controls $(p=0.31)$. A homozygous p.R78Q-SULT1A1 variant was identified in a 2-month-old male SIDS case and a hemizygous p.V37I-MAOA variant was identified in a 3.8-month-old male SIDS case. In European controls, there was a homozygous p.R297Q-MAOA variant in one control and a homozygous p.V231I-CHRM2 variant in a second control; a third control hosted compound heterozygous HADHA variants (p.K249N and p.E510Q; see Online Supplement Tables 5 and 6). All of the variants were classified as a VUS except for the p.E510Q-HADHA variant, which was classified as pathogenic. The p.E510-HADHA variant has been reported previously in both homozygous and compound heterozygous cases in a large number of individuals and families with longchain 3-hydroxyacyl-CoA dehydrogenase (LCHAD) deficiency and demonstrated to result in significant loss of enzyme activity with this variant. ${ }^{28}$

There were no significant differences in the yield of dominant/recessive NSVs among all 61 SIDS-susceptibility genes when comparing sex, sleep position (supine vs. prone), or cosleeping (yes vs. no) (Table 3).

\section{SIDS biological pathway gene-collapsing analysis}

A rare variant, gene-collapsing burden analysis performed on gene sets involving eight different biological pathways (genetic heart disease, epilepsy, inborn errors of metabolism,
Table 3 The effect of various demographics on the yield of ultrarare gene variants in SIDS cases

\begin{tabular}{llll} 
Demographic & & Overall $(n=278)$ & $p$ value \\
\hline Sex & Male & $28 / 173(16.2 \%)$ & 1.0 \\
\multirow{2}{*}{ Age } & Female & $17 / 105(16.2 \%)$ & \\
\multirow{3}{*}{ Sleep position } & $2-4$ months & $22 / 154(14.3 \%)$ & 0.33 \\
& Other & $23 / 124(18.5 \%)$ & \\
& Prone & $5 / 52(9.6 \%)$ & 0.40 \\
& Supine & $14 / 85(16.5 \%)$ & \\
& Side & $4 / 29(13.4 \%)$ & \\
& Seated & $0 / 2(0.0 \%)$ & \\
Cosleeping & Unknown & $22 / 110(20.0 \%)$ & \\
& Yes & $10 / 66(15.2 \%)$ & 0.24 \\
& No & $13 / 106(12.3 \%)$ & \\
& Unknown & $22 / 106(20.8 \%)$ & \\
\hline
\end{tabular}

respiratory control, other neurological conditions, autonomic nervous system, immune system, and nicotine metabolizing) previously implicated in SIDS pathogenesis also failed to yield any significant associations (Table 4).

\section{DISCUSSION}

Since the proposal of the "triple-risk hypothesis" over 20 years ago, investigators have been searching for monogenetic explanations as a substrate for infant vulnerability to SIDS. Some of the suspected sources of an infant's "underlying vulnerability" include genetic determinants leading to dysfunction of the central and autonomic nervous system, inborn errors of metabolism, and cardiac channelopathies (Supplemental Reference list). While there have been no clear diagnostic markers identified, several common polymorphisms have been identified to be significantly overrepresented in distinct SIDS ethnic populations. ${ }^{8}$

Recently, we completed exome sequencing-based molecular autopsy with a genetic heart disease (GHD) gene-specific analysis for 278 unrelated European ancestry SIDS cases to determine the contribution of monogenic heart disease to 


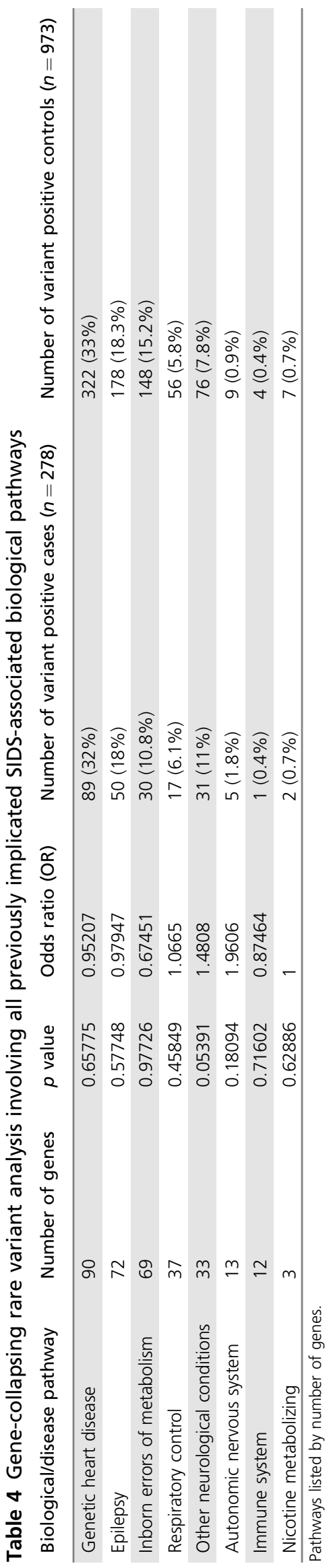

SIDS pathology. Less than $12 \%$ of the European SIDS cases hosted an ultrarare (MAF <0.005\%) "potentially informative" variant in one of the 90 GHD-susceptibility genes analyzed. However, according to the ACMG guidelines only $4.3 \%$ of the cases possessed immediately clinically actionable GHD-associated variants (i.e., pathogenic or likely pathogenic).

Our current study now examines the potential contribution of noncardiac genes in the pathogenesis of SIDS using a similar approach to examine 61 published noncardiac genes previously implicated in SIDS. ${ }^{8,17-21}$ The majority had been identified as potential "SIDS-susceptibility" genes following both common and rare variant association studies, typically involving promoter region variants. However, only approximately $55 \%$ had been associated previously with either a dominant or recessive rare monogenic disease. Although $28(46 \%)$ have never been associated with any monogenic disorder (dominant or recessive) and 18 (29.5\%) have only been associated with recessive disease, we chose to interrogate all 61 genes under both dominant and recessive inheritance models to examine the potential role of each gene for its involvement in the monogenic basis for SIDS.

Soberingly, there was no significantly increased burden of ultrarare variants in all 61 genes in SIDS cases compared with ethnically matched controls ( 15.5 vs. $11.7 \%, p=0.10)$ in a dominant inheritance model or rare homozygous/compound heterozygous variants in cases over controls using a recessive inheritance model ( 0.72 vs. $0.3 \%, p=0.31)$. In addition, there was a negligible yield of immediately clinically actionable disease-associated variants (i.e., pathogenic or likely pathogenic) in SIDS cases $(0.36 \%)$ and controls $(0.31 \%)$ with no significant difference detected. Furthermore, there was no difference in yield of variants between cases and controls for 59 of the 61 genes when analyzed independently.

Only two genes (ECE1 and SLC6A4) achieved the $p<0.05$ threshold; however ultrarare SNVs in both genes may also be irrelevant because it would be predicted that perhaps 3 of the 61 genes would achieve this cut-off by chance alone. The ECE1 encoded-endothelin-converting enzyme 1 (ECE1) has been associated previously with autonomic dysfunction and has been proposed to play a potential role in SIDS susceptibility. In 1999, a heterozygous loss-of-function ECE1 variant (p.C742R), absent in 100 controls, was identified in a single patient with Hirschsprung disease, structural cardiac defects, craniofacial abnormalities, other dysmorphic features, and autonomic dysfunction. ${ }^{29}$ In 2004, Weese-Mayer and colleagues reported the identification of a single ECE1 missense variant (p.T354A) in 1 of 46 black SIDS cases that was absent amongst 46 ethnically matched controls. ${ }^{30}$ However, both of these variants have now been observed within the gnomAD database at a MAF that would suggest that they may be too common (p.C742R present in $48 / 62,405$ [0.08\%] European individuals; p.T354A present in 106/12,015 [0.89\%] African individuals) to be responsible for the disease phenotypes observed originally. 
Solute carrier family 6 member 4 (SLC6A4) gene encodes for the serotonin transporter 1 (5-HTT). SLC6A4 missense variants have not previously been directly linked with SIDS, however there have been other associations between SIDS and SLC6A4. A number of studies have explored the association of SIDS with two common functional insertion/deletion polymorphisms thought to influence SLC6A4 gene expression; one in the promoter region (5-HTT promoter polymorphism or 5 -HTTLPR) and one in the second intron. Initial results showed an increased frequency in the long (L) allele of the SLC6A4 promoter region in SIDS victims and infants with apparent life-threatening events as well as an association with the intron 2 polymorphism and SIDS. However, several studies involving larger SIDS cohorts failed to replicate these early findings. ${ }^{12,31,32}$ In our SIDS cohort we identified two ultrarare missense variants, V524M-SLC6A4 and A228DSLC6A4, in two separate female 2-month-old SIDS victims, both of which were classified as VUS by ACMG criteria. This is an interesting finding in our study, though the result is only borderline for "statistical" significance $(p=-.049)$. Therefore, further functional data would be required before attributing any potential contribution of SLC6A4 genetic variation in the pathogenesis of SIDS.

Given the prior inconsistent and weak associations between both ECE1 and SLC6A4 variants in SIDS, it would be way too premature to conclude that ultrarare nonsynonymous ECE1 and SLC6A4 variants are contributing factors to infant vulnerability for SIDS. In fact, based on our analysis, we suggest that many of the previously established SIDSsusceptibility genes should be reconsidered and potentially reclassified to "limited evidence" or "refuted evidence" disease-gene designations. Replication of these results in other large SIDS cohorts and functional data are necessary before concluding that $1-2 \%$ of SIDS cases may stem from nonsynonymous ECE1 and SLC6A4 variants.

Following our recent SIDS case-control gene-collapsing ultrarare variant burden analysis involving the four major cardiac channelopathy genes (KCNQ1, KCNH2, SCN5A, and $R Y R 2)$, we extended our case-control gene-collapsing burden analysis to include 331 genes thought to be important in 8 different candidate biological pathways previously implicated in SIDS. While the gene-collapsing analysis did not identify any specific biological pathways to be significantly associated with SIDS, the association between "other neurological genes" was borderline for statistical significance $(p=0.05)$, suggesting this may be an important pathway for SIDS pathology. In fact, for one of the other neurological genes, $S C N 4 A$, we recently demonstrated a significant $(p=0.0057)$ overrepresentation of functionally disruptive variants in European SIDS versus ethnic-matched controls. ${ }^{33}$ The SCN4A encoded skeletal muscle voltage-gated sodium channel (Nav1.4) is important in controlling skeletal respiratory muscle contraction. Interestingly, we did not find an association between specific epilepsy genes on gene-collapsing burden analysis ( $18 \%$ in cases vs. $8.3 \%$ in controls, $p=0.58)$ despite other groups recently demonstrating an association with epilepsy variants and SIDS, particularly SCN1A in those infants with hippocampal abnormalities. ${ }^{34}$

Investigation of all previously implicated noncardiac, SIDSsusceptibility genes in a large European SIDS case-control analysis has failed to show any significant associations of ultrarare or novel variation consistent with autosomal dominant and recessive inheritance patterns. Furthermore, there are few pathogenic or likely pathogenic variants. This demonstrates clearly that there is very little monogenic disease involving these specific genes underlying SIDS, at least within their translated open reading frames and canonical splice sites.

Whether or not an unbiased analysis of the open reading frames/canonical splice sites of all 20,000 + genes will reveal any novel monogenic substrates for infant vulnerability remains to be determined. It also remains to be seen whether more common genetic variation may associate with infant vulnerability to sudden death thereby supporting a complex polygenic inheritance model for infant vulnerability. In the interim, these previously implicated noncardiac SIDSsusceptibility genes should be demoted to "limited evidence" genes at least in terms of a penetrant, monogenic basis for SIDS.

\section{ELECTRONIC SUPPLEMENTARY MATERIAL}

The online version of this article (https://doi.org/10.1038/s41436018-0131-4) contains supplementary material, which is available to authorized users.

\section{ACKNOWLEDGEMENTS}

The authors gratefully acknowledge both the medical examiners and coroners in both the United Kingdom and the United States for referring the sudden death victims to our programs in an effort to find an explanation for the infant's sudden death. This work was supported by Eunice Kennedy Shriver National Institute of Child Health \& Human Development of the National Institutes of Health (R01HD042569 to M.J.A.) and by the British Heart Foundation ([L.W. and E.R.B.] BHF Clinical Research Training Fellowship FS/13/78/30520). The content is solely the responsibility of the authors and does not necessarily represent the official views of the National Institutes of Health. D.J.T. and M.J.A. are also supported by the Mayo Clinic Windland Smith Rice Comprehensive Sudden Cardiac Death Program. B.G. is supported by a National Health and Medical Research Council (NHMRC) Early Career Fellowship (1122330). L.W. was also supported by additional funds from Biotronik and Cardiac Risk in the Young. E.R.B. is supported by the Higher Education Funding Council for England and receives funds from The Robert Lancaster Memorial Fund sponsored by McColl's Retail Group Ltd. The establishment of the Edinburgh SIDS DNA cohort was supported by a grant from Newlife Foundation.

\section{DISCLOSURE}

M.J.A. is a consultant for Audentes Therapeutics, Boston Scientific, Gilead Sciences, Invitae, Medtronic, MyoKardia, and St. Jude Medical. M.J.A. and Mayo Clinic have an equity/royalty- 
based relationship (without remuneration so far) with AliveCor, Blue Ox Health Corporation, and StemoniX. However, none of these entities were involved in this study in any way. The other authors declare no conflicts of interest.

\section{REFERENCES}

1. Krous HF, Beckwith JB, Byard RW, et al. Sudden infant death syndrome and unclassified sudden infant deaths: a definitional and diagnostic approach. Pediatrics. 2004;114:234-238.

2. Kinney HC, Richerson GB, Dymecki SM, et al. The brainstem and serotonin in the sudden infant death syndrome. Annu Rev Pathol. 2009:4:517-550.

3. Moon RY, Horne RS, Hauck FR. Sudden infant death syndrome. Lancet. 2007;370:1578-1587

4. Matthews TJ, MacDorman MF. Infant mortality statistics from the 2010 period linked birth/infant death data set. Natl Vital Stat Rep. 2013;62:1-26.

5. Patel V. Unexplained deaths in infancy, England and Wales: 2015. 2015. http://www.ons.gov.uk/peoplepopulationandcommunity/birthdeathsand marriages/death/bulletins/unexplaineddeathsininfancyenglandandwales/ 2015. Accessed 4 May 2018.

6. Eunice Kennedy Shriver National Institute of Child Health and Human Development, NIH, DHHS. (2000). From Cells to Selves: NICHD Strategic Plan (N/A). Washington, DC: U.S. Government Printing Office.

7. Filiano JJ, Kinney HC. A perspective on neuropathologic findings in victims of the sudden infant death syndrome: the triple-risk model. Biol Neonate. 1994;65:194-197.

8. Salomonis N. Systems-level perspective of sudden infant death syndrome. Pediatr Res. 2014;76:220-229.

9. Schwartz PJ. Cardiac sympathetic innervation and the sudden infant death syndrome. A possible pathogenetic link. Am J Med. 1976;60:167-172.

10. Guntheroth WG. Theories of cardiovascular causes in sudden infant death syndrome. J Am Coll Cardiol. 1989;14:443-447.

11. Yun AJ, Lee PY. Sudden death among infants and adults: companion disorders of maladaptive sympathetic bias. Med Hypotheses. 2004;62:857-860.

12. Weese-Mayer DE, Ackerman MJ, Marazita ML, et al. Sudden infant death syndrome: review of implicated genetic factors. Am J Med Genet A. 2007:143A:771-788.

13. Van Norstrand DW, Ackerman MJ. Genomic risk factors in sudden infant death syndrome. Genome Med. 2010;2:86.

14. Ferrante $L$, Rognum TO, Vege $A$, et al. Altered gene expression and possible immunodeficiency in cases of sudden infant death syndrome. Pediatr Res. 2016:80:77-84.

15. Tester DJ, Wong LC, Chanana P, et al. Cardiac genetic predisposition in sudden infant death syndrome. J Am Coll Cardiol. 2018;71:1217-1227

16. Ruark $E$, Münz $M$, Renwick $A$, et al. The ICR1000 UK exome series: a resource of gene variation in an outbred population [version 1; referees: 3 approved]. F1000Research 2015:4:883. https://doi.org/10.12688/ f1000research.7049.1.

17. Cutz E. Whole exome sequencing reveals CLCNKB mutations in a case of sudden unexpected infant death. Pediatr Dev Pathol. 2015;18:339-340.
18. Fard D, Laer K, Rothamel T, et al. Candidate gene variants of the immune system and sudden infant death syndrome. Int J Legal Med. 2016;130:1025-1033.

19. Laer K, Dork T, Vennemann M, Rothamel T, Klintschar M. Polymorphisms in genes of respiratory control and sudden infant death syndrome. Int $J$ Legal Med. 2015;129:977-984.

20. Lahrouchi $N$, Lodder EM, Mansouri M, et al. Exome sequencing identifies primary carnitine deficiency in a family with cardiomyopathy and sudden death. Eur J Hum Genet. 2017;25:783-787.

21. Yamamoto $\mathrm{T}$, Tanaka H, Emoto $\mathrm{Y}$, et al. Carnitine palmitoyltransferase 2 gene polymorphism is a genetic risk factor for sudden unexpected death in infancy. Brain Dev. 2014;36:479-483.

22. Richards S, Aziz N, Bale $S$, et al. Standards and guidelines for the interpretation of sequence variants: a joint consensus recommendation of the American College of Medical Genetics and Genomics and the Association for Molecular Pathology. Genet Med. 2015; 17:405-424.

23. Li Q, Wang K. InterVar: clinical interpretation of genetic variants by the 2015 ACMG-AMP guidelines. Am J Hum Genet. 2017; 100:267-280.

24. Kircher M, Witten DM, Jain P, O'Roak BJ, Cooper GM, Shendure J. A general framework for estimating the relative pathogenicity of human genetic variants. Nat Genet. 2014:46:310-315.

25. Frigeni $M, B a l a k r i s h n a n ~ B, Y i n ~ X$, et al. Functional and molecular studies in primary carnitine deficiency. Hum Mutat. 2017;38:1684-1699.

26. Lemke JR, Geider K, Helbig KL, et al. Delineating the GRIN1 phenotypic spectrum: a distinct genetic NMDA receptor encephalopathy. Neurology. 2016:86:2171-2178.

27. Fry $A E$, Fawcett $K A$, Zelnik $N$, et al. De novo mutations in GRIN1 cause extensive bilateral polymicrogyria. Brain 2018:141:698-712.

28. Olpin SE, Clark S, Andresen BS, et al. Biochemical, clinical and molecular findings in LCHAD and general mitochondrial trifunctional protein deficiency. J Inherit Metab Dis. 2005;28:533-544.

29. Hofstra RM, Valdenaire O, Arch E, et al. A loss-of-function mutation in the endothelin-converting enzyme 1 (ECE-1) associated with Hirschsprung disease, cardiac defects, and autonomic dysfunction. Am J Hum Genet. 1999;64:304-308.

30. Weese-Mayer DE, Berry-Kravis EM, Zhou L, et al. Sudden infant death syndrome: case-control frequency differences at genes pertinent to early autonomic nervous system embryologic development. Pediatr Res. 2004:56:391-395.

31. Weese-Mayer DE, Berry-Kravis EM, Maher BS, Silvestri JM, Curran ME, Marazita ML. Sudden infant death syndrome: association with a promoter polymorphism of the serotonin transporter gene. Am J Med Genet A. 2003;117A:268-274.

32. Filonzi L, Magnani $C$, Nosetti L, et al. Serotonin transporter role in identifying similarities between SIDS and idiopathic ALTE. Pediatrics. 2012;130:e138-144

33. Mannikko. RWL, Tester. D, Thor MG, et al. Dysfunction of the skeletal muscle gated sodium channel in sudden infant death syndrome. Lancet. 2018;391:1483-1492.

34. Brownstein CA, Goldstein RD, Thompson $\mathrm{CH}$, et al. SCN1A variants associated with sudden infant death syndrome. Epilepsia. 2018;59: e56-e62.

Belinda Gray, MBBS PhD ${ }^{1,2,3,4}$, David J. Tester, BS ${ }^{5}$, Leonie CH Wong, MBBChir ${ }^{1,2}$, Pritha Chanana, $\mathrm{MS}^{6}$, Amie Jaye, $\mathrm{MSc}^{7}$, Jared $\mathrm{M}$. Evans, $\mathrm{MS}^{6}$, Alban-Elouen Baruteau, MD PhD ${ }^{1,2,8}$, Margaret Evans, $\mathrm{MBChB}^{9}$, Peter Fleming, $\mathrm{PhD}^{10}$, Iona Jeffrey, $\mathrm{MBChB}{ }^{11,12}$, Marta Cohen, $\mathrm{MD}^{13}$, Jacob Tfelt-Hansen, MD DMSc ${ }^{14,15}$, Michael A. Simpson, PhD ${ }^{7}$, Michael J. Ackerman, MD PhD ${ }^{5}$ and Elijah R. Behr, MD ${ }^{1,2}$

${ }^{1}$ Molecular and Clinical Sciences Research Institute, St George's University of London, London, United Kingdom. ${ }^{2}$ Cardiology Clinical Academic Group, St George's University Hospitals' NHS Foundation Trust, London, United Kingdom. ${ }^{3}$ Agnes Ginges Centre for Molecular Cardiology, Centenary Institute, Sydney, Australia. ${ }^{4}$ Sydney Medical School, University of Sydney, Sydney, Australia. ${ }^{5}$ Departments of Cardiovascular Medicine (Division of Heart Rhythm Services), Pediatrics (Division of Pediatric Cardiology), and 
Molecular Pharmacology \& Experimental Therapeutics (Windland Smith Rice Sudden Death Genomics Laboratory), Mayo Clinic, Rochester, Minnesota, USA. ${ }^{6}$ Department of Health Sciences Research, Mayo Clinic, Rochester, Minnesota, USA. ${ }^{7}$ Medical and

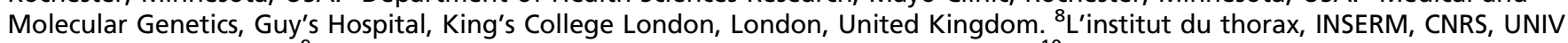
Nantes, Nantes, France. ${ }^{9}$ Royal Infirmary of Edinburgh, Edinburgh, United Kingdom. ${ }^{10}$ Centre for Child and Adolescent Health, Bristol Medical School, University of Bristol, Bristol, United Kingdom. ${ }^{11}$ Department of Cellular Pathology, St George's University of London, London, United Kingdom. ${ }^{12}$ Department of Cellular Pathology' St George's University Hospitals' NHS Foundation Trust, London, United Kingdom. ${ }^{13}$ Histopathology Department, Sheffield Children's Hospital NHS FT, Sheffield, United Kingdom.

${ }^{14}$ Department of Cardiology, The Heart Centre, Copenhagen University Hospital, Rigshospitalet, Copenhagen, Denmark.

${ }^{15}$ Department of Forensic Medicine, Faculty of Medical Sciences, University of Copenhagen, Copenhagen, Denmark 